\title{
The star formation history of the LSB galaxy UGC 5889^
}

\author{
A. Vallenari ${ }^{1}$, L. Schmidtobreick ${ }^{2}$, and D. J. Bomans ${ }^{3}$ \\ 1 Astronomical Observatory of Padova, Vicolo dell' Osservatorio 5, 35122 Padua, Italy \\ 2 European Southern Observatory, Casilla 19001, Santiago 19, Chile \\ 3 Astronomisches Institut der Ruhruniversität Bochum, 44780 Bochum, Germany \\ e-mail: vallenari@astrpd.pd.astro.it
}

Received 21 April 2004 / Accepted 24 January 2005

\begin{abstract}
We present HST photometry of the LSB galaxy UGC 889 and derive its recent star formation history. In the last $200 \mathrm{Myr}$ the star formation proceeded in modest bursts at a rate of the order of $10^{-2}-10^{-3} M_{\odot} / \mathrm{yr}$, with periods of extremely low SFR or even quiescence. The rate derived from the present study for the last $20 \mathrm{Myr}$ is in agreement with the $\mathrm{H}_{\alpha}$ emission from the galaxy. The presence of a consistent population older than $200 \mathrm{Myr}$ is suggested by the data. However, observational errors and completeness correction prevent any firm conclusion on the oldest age. The total mass of stars is of the order of $5.5 \times 10^{7} M_{\odot}$. Even if the recent episodes of star formation have heated the gas and carved a hole in the disk, blow-away of the gas is unlikely to occur.
\end{abstract}

Key words. stars: formation - galaxies: stellar content - ISM: bubbles - stars: AGB and post-AGB

\section{Introduction}

It is well known that low surface brightness (LSB) galaxies contribute significantly to the total galaxy counts (see e.g. Marzke \& da Costa 1997) and might even constitute the majority of galaxies (O'Neil \& Bothun 2000). LSB galaxies show a large variety of properties, ranging from the blue to the red end of galaxy colours (O'Neil et al. 1997, 2000) and consisting of dwarfs as well as of massive systems (Sprayberry et al. 1995). Concerning their metal content, the vast majority of LSB galaxies show low metal content (de Block \& van der Hulst 1998), although some red LSB galaxies having solar metallicity have been recently detected (Bergmann et al. 2003). Among these diverse systems, we focus on one of the most common types, the blue LSB (BLSB) galaxies (see the review by Impey \& Bothun 1997 and references therein), late-type disk galaxies having B surface brightness $>23$ mag $\operatorname{arcsec}^{-2}$ where colours of $(B-V)<0.6$ are found (O'Neil et al. 1997). These blue colours are puzzling primarily because of the restrictions they put on the star formation history of LSB galaxies. Even if they present low surface gas density, BLSB are among the most gas-rich galaxies at a given total luminosity. The high gas fraction of BLSB galaxies, together with their low metal content, suggest that they are relatively unevolved objects. This can be interpreted in various ways. For instance BLSB galaxies can have very low present and past star formation rates (SFR): in these systems the main phase of star formation is still to occur. Alternatively, these galaxies can have a stellar population similar to those of high surface brightness spirals, but their

\footnotetext{
* Based on observations collected with Hubble Space Telescope.
}

luminosity can be dominated by a young population. Until now, the investigation of the stellar content of LSB galaxies has mainly been based on the discussion of the integrated colours. Many studies are consistent with a scenario where BLSB galaxies have on average a constant or increasing SFR with a ratio of young to old stars larger than found in high brightness spirals (van den Hoek et al. 2000; Knezek 1993; Bell \& de Jong 1999). However, different opinions can be found in the literature, either suggesting that the SFR can be proceeding in frequent, small amplitude bursts (van den Hoek et al. 2000) or that the bulk of the population is older than 5-7 Gyr (Padoan et al. 1997). Finally, it is far from being understood how stars form in a low density, low metal content environment and what is the relation between SFR and other properties of LSB galaxies (i.e. the angular momentum) (see among others O'Neil et al. 2000; Boissier et al. 2003; Dalcanton et al. 1997).

In this paper we focus on the study of a resolved LSB galaxy: UGC 5889 (NGC 3377A). This dwarf galaxy is a member of the Leo I group. Due to its similar redshift and resulting proximity - only $20 \mathrm{kpc}$ in projected distance (Sandage \& Hoffman 1991), it is thought to be a companion of the large elliptical NGC 3377. With $M_{\mathrm{H}_{\mathrm{I}}} / L_{B} \sim 0.3 M_{\odot} / L_{\odot}$, it is gas rich (Knezek et al. 1999). It is classified as a low surface brightness dwarf having an average surface brightness inside the Holmberg radius of $B=23.97 \mathrm{mag} \operatorname{arcsec}^{-2}$. Moderately blue colours $(U-B)=0.06 \pm 0.05$, and $(B-V)=0.64 \pm 0.06$ are given by Prugniel \& Heraudeau (1998).

Base on its optical morphology, Knezek et al. (1999) suggested the presence of a spiral structure classifying the galaxy as $\mathrm{SABm}$. This has recently been supported by high resolution 


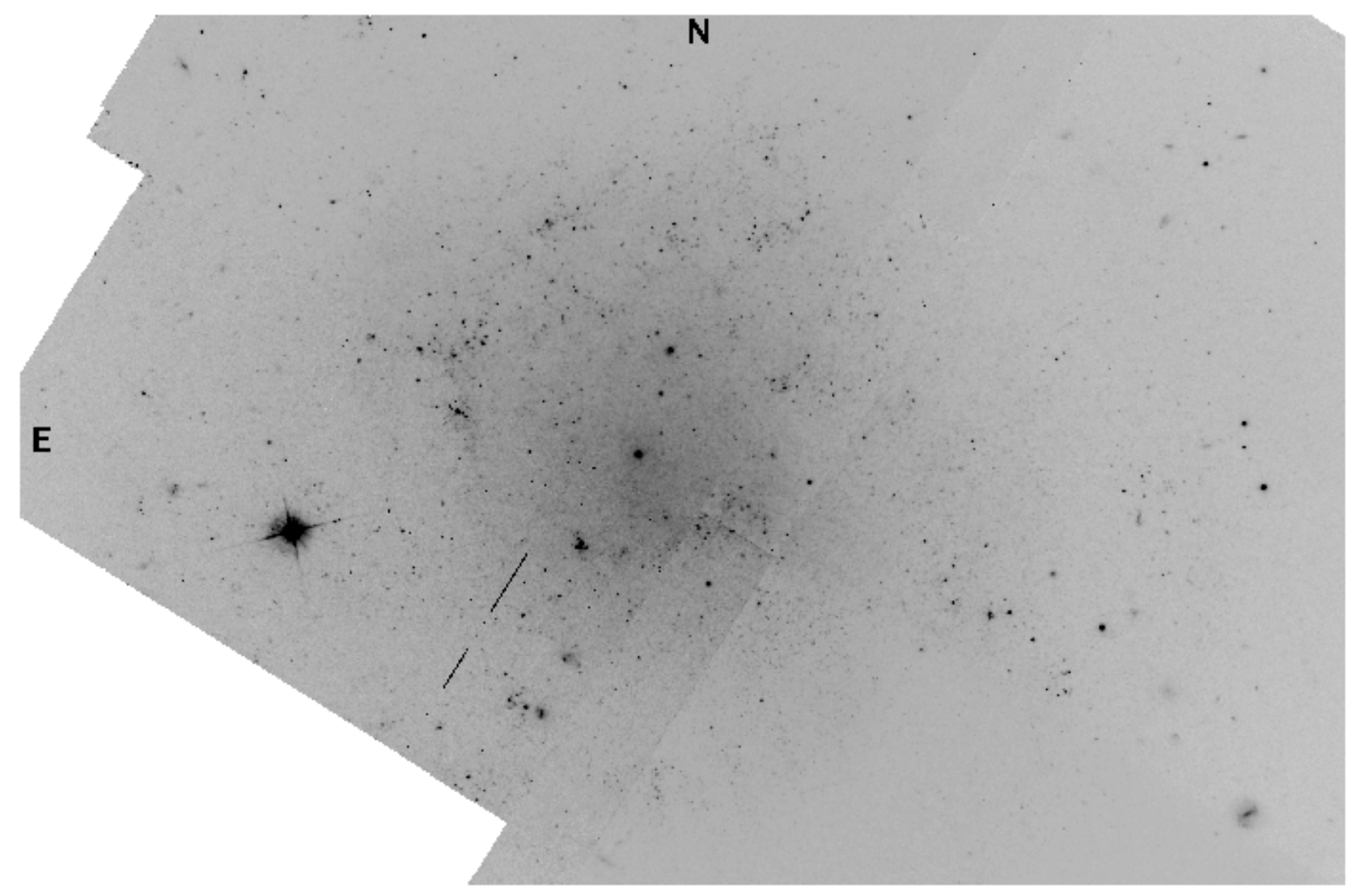

Fig. 1. Composite image of the central area of the galaxy UGC 5889.

spectroscopic H I-measurements of Huchtmeier et al. (2003), who find a double peaked profile for this galaxy which is typical for spirals. The H I intensity maps of Simpson \& Gottesman (2000) show a ring-shaped distribution of the gas which is surrounding the bulk of the optical body and is correlated with the young star groups discovered by Vicari et al. (2002).

Knezek et al. (1999) also measured the $\mathrm{H}_{\alpha}$ luminosity of UGC 5889 and found a relatively low value of $L_{\mathrm{H}_{\alpha}} \sim 2.6 \times$ $10^{38} \mathrm{ergs} \mathrm{s}^{-1}$, which is about ten times lower than the average $\mathrm{H}_{\alpha}$ luminosity in the sample of quiescent dwarf spiral galaxies studied by van Zee et al. (1997). Thus, despite the dense environment where it is located, UGC 5889 appears to be a very quiet dwarf galaxy. Knezek et al. (1999) discuss the possibility that it might represent a true transition object between late type dwarf galaxies and low SFR dwarf ellipticals as suggested by Sandage \& Hoffman (1991). However they reject the hypothesis, since with its large gas reservoir UGC 5889 can continue to form stars at the present SFR for much longer than the Hubble time.

The star formation history of UGC 5889 is unknown. It is still not clear whether the current SFR represents the mean level over the galaxy lifetime or, whether the star formation of this galaxy has occurred at a different rate in the past. This paper deals with the determination of the SFR and the SFR history from the analysis of colour-magnitude diagrams (CMDs). The plan of this paper is as follows: in Sect. 2, we discuss the observations, the data reduction and we present the observed CMD. In Sect. 3, we derive the distance modulus, in Sect. 4, the basic CMD features. In Sect. 5 we determine the star formation history of the galaxy and we discuss the feedback from star formation. In Sect. 6, we compare the SFR of UGC 5889 with other LSB galaxies. Conclusions are drawn in Sect. 7.

\section{Observation and reduction}

We use HST archive data of UGC 5889 taken in 1995 with $W F P C 2$ at two different position angles and locations. In total, 21 partially overlapping $F 555 \mathrm{~W}$ and four $F 814 \mathrm{~W}$ frames are used including long and short exposed frames and yielding a total exposure time of $6000 \mathrm{~s}$ in $F 814 \mathrm{~W}$ and $25300 \mathrm{~s}$ in $F 555 \mathrm{~W}$.

A composite image of the galaxy in the F555W filter is presented in Fig. 1. It shows the high spatial resolution, which allowed the photometric measurement of individual stars.

The data have been reduced using the stand-alone version of DAOPHOT including ALLFRAME and following the ALLFRAME cookbook (Turner 1996). To de-select suspected clusters and foreground galaxies we excluded the objects with FWHM larger than 2 pixels using the ALLFRAME parameter "sharp". In total, we derive photometry for 4843 stars in the galaxy spanning the $F 814 \mathrm{~W}$ magnitude range from $20^{\mathrm{m}}$ to fainter then $27^{\mathrm{m}}$.

The photometric calibration has been done following the prescriptions by Dolphin (2000). We estimate the error on the zero point as $0.1 \mathrm{mag}$. The completeness factors $\Lambda$ are calculated as usual by means of artificial star experiments and are 


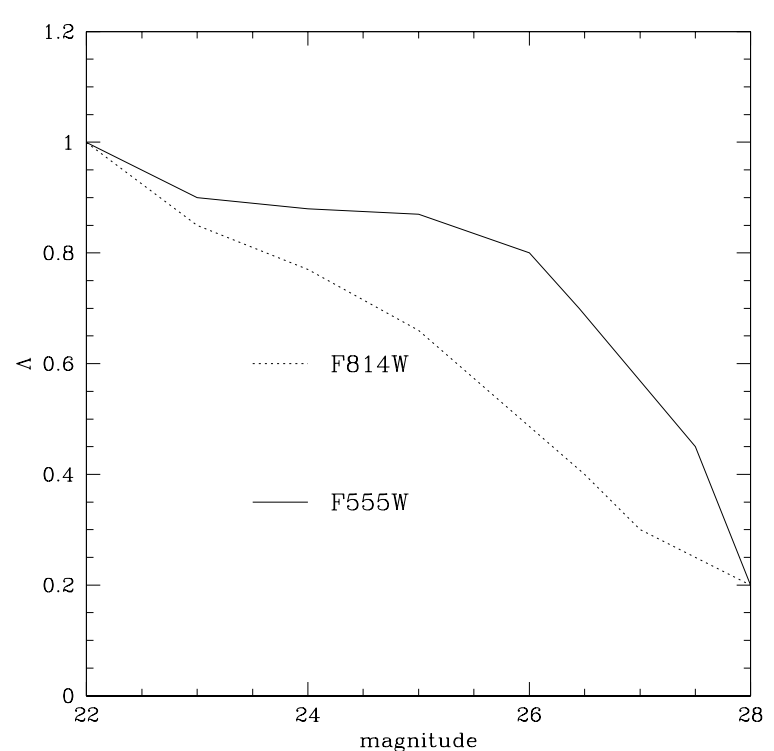

Fig. 2. Completeness factors $\Lambda$ for $F 555 W$ and $F 814 W$ magnitudes.

Table 1. The photometric errors as a function of the magnitude.

\begin{tabular}{lccccc}
\hline \hline$F 555 W[\mathrm{mag}]$ & $<24$ & 25 & 26 & 27 & 27.5 \\
$\sigma_{\text {phot }}[\mathrm{mag}]$ & $<0.1$ & 0.1 & 0.2 & 0.3 & 0.45 \\
\hline$F 814 W[\mathrm{mag}]$ & $<24$ & 25 & 26 & & \\
$\sigma_{\text {phot }}[\mathrm{mag}]$ & $<0.1$ & 0.2 & 0.3 & & \\
\hline
\end{tabular}

plotted in Fig. 2. The data are complete at $40 \%$ level for magnitudes brighter than $F 555 W<27.5$ or $F 814 W<26.5$. Artificial star experiments are used as well to derive the errors on the magnitudes due both to the Poisson noise and crowding. These errors are listed in Table 1. Photometric errors due only to the Poisson statistics on the noise are estimated by ALLFRAME. As expected, they are smaller, being $<0.1,0.14,0.22,0.30$ for $F 555 W$ magnitudes $<25,26,27,27.5$, respectively. The analogous photometric errors on $F 814 W$ magnitude are $<0.1,0.12$, 0.24 for $F 814 W=24,25,26$ respectively.

The CMD of the whole galaxy is plotted in Fig. 3. The blue plume is located at $(F 555 W-F 814 W)<0.5$ and extends from the limiting magnitude at $F 814 W \sim 27$ up to 22 . The red evolved stars extend from $F 814 W \sim 27$ to $\sim 23$ and have $(F 555 W-F 814 W)>0.5$.

\section{Distance modulus determination}

The I magnitude of the tip of the red giant branch (TRGB) has been shown to be an excellent distance indicator for nearby resolved galaxies (Lee et al. 1993). The position of the TRGB in UGC 5889 has been obtained dividing the observational $F 814 W-(F 555 W-F 814 W)$ CMD into small intervals of width $(0.15,0.15)$ and then convolving the bi-dimensional histogram with an edge detector function, namely a gradient filter with an angle of 90 degrees, increasing the contrast at increasing magnitudes. The sharp cut-off at the end of the RGB is clearly detected by the gradient filter (see Fig. 4). We take the position of the main peak as our estimate of the tip magnitude and the half width at half maximum of the peak as the

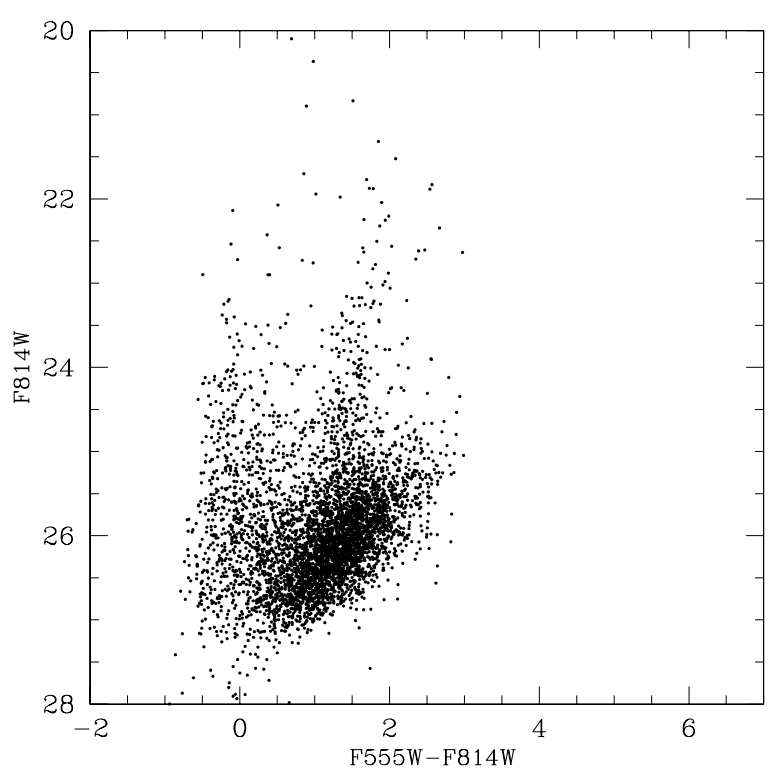

Fig. 3. CMD of UGC 5889 (whole field).

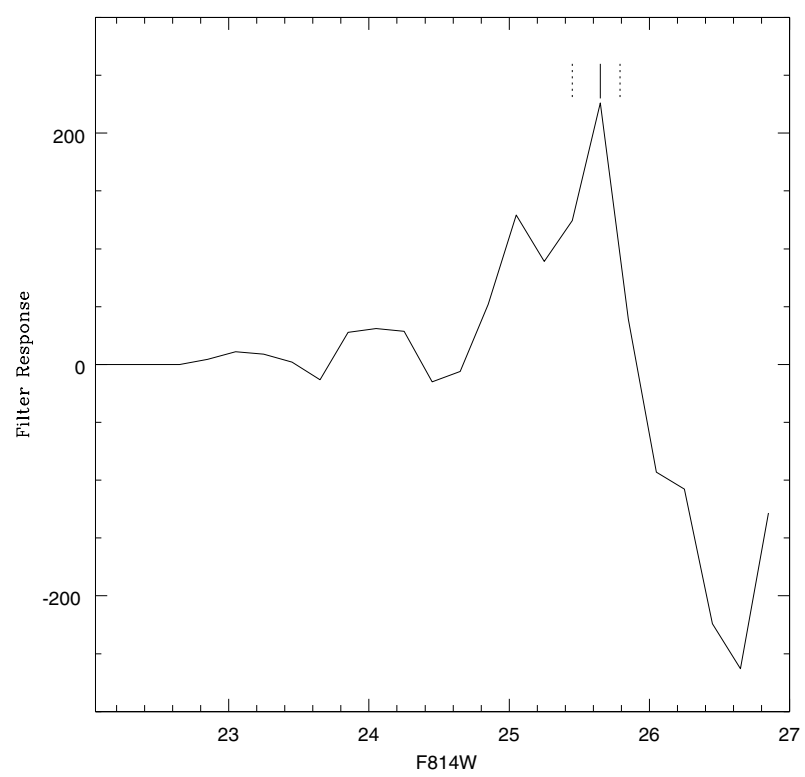

Fig. 4. RGB tip detection: the gradient filter response to the luminosity function is plotted as a function of the magnitude(see text for more detail). The position of the main peak is taken as indication of the RGB tip (solid line). The dotted lines show the half width at half maximum of the peak taken as estimate of the uncertainties on the tip detection.

uncertainty on the determination. We find $F 814 W=25.6 \pm 0.2$ as the TRGB magnitude. Since all the existing calibrations of the TRGB magnitude are expressed in the Johnson passbands, we first convert the de-reddened HST F555, F814 magnitudes to the $V, I$ magnitudes.

We derive the foreground reddening from the maps of Schlegel et al. (1998), $E_{B-V}=0.034$, resulting in $E_{F 555 W-F 814 W}=0.06$. This value is slightly lower than the $E_{B-V}=0.06$ estimated by Knezek et al. (1999) using the standard dust-to-gas ratio of Diplas \& Savage (1994) and the HI measurements of Hartmann \& Burton (1997). 
The resulting difference in magnitude is within the photometric errors and hence does not affect our further conclusions.

We therefore use $E_{(B-V)}=0.034$ corresponding to $E_{(F 555-F 814)}=0.06$ and $A_{F 814}=0.09$. With these values, the magnitude and colour at the TRGB transform into the Johnson $I_{0}=25.4,(V-I)_{0}=1.48$.

The distance modulus is derived from the $I_{\mathrm{TRGB}}$ magnitude at the TRGB using the definition:

$(m-M)_{0}=I_{\mathrm{TRGB}}-M_{I, \mathrm{TRGB}}$.

The dependence of $M_{I}^{\mathrm{TRGB}}$ on the metal content $[\mathrm{M} / \mathrm{H}]$ is given by the recent calibrations of Bellazzini et al. (2001, 2004):

$M_{I}^{\mathrm{TRGB}}=0.258[\mathrm{M} / \mathrm{H}]^{2}+0.676[\mathrm{M} / \mathrm{H}]-3.626$.

This holds true for populations in the age range from 2 to 12 Gyr and $-1.5<[\mathrm{M} / \mathrm{H}]<-0.6$. This method to derive the distance has been proved to be independent of the details of the star formation history (Barker et al. 2004). In the above metallicity range, $M_{I}^{\mathrm{TRGB}}=-4.05$ when $[\mathrm{M} / \mathrm{H}]=-1.5$ and $M_{I}^{\mathrm{TRGB}}=-3.91$ when $[\mathrm{M} / \mathrm{H}]=-0.6$. Since the metal content of UGC 5889 is likely inside the above range (see following section), we are probably not seriously in error if we assume $M_{I, \mathrm{TRGB}}=-3.98 \pm 0.07$. This results in a distance modulus of $(m-M)_{0}=29.4 \pm 0.2$, where the quoted errors take into account both the uncertainties on the absolute magnitude and on the observational magnitude of the tip. This value is in reasonable agreement with previous determinations. For the companion NGC 3377, Graham et al. (1997) derive a distance modulus $30.01 \pm 0.15$. A direct determination for UGC 5889 has been made by Makarova \& Karachentsev (1998) using the magnitude of the brightest blue stars, and they find $(m-M)_{0}=29.8$.

\section{CMD discussion}

To derive information about the SFR, we compare the CMD with isochrones of different metallicity. Figure 5 shows the CMD with the isochrones of $Z=0.008$ which reproduce the main features of the CMD such as the slope of the giant branch and the location of those stars brighter than $F 814 W \sim 26$ and bluer than $(F 555 W-F 814 W) \sim 0.5$ (blue plume). This blue plume is formed by a mixture of main sequence and blue loop stars which crowd the same CMD region.

A solar metallicity is only marginally consistent, predicting a colour of the core He-burning stars redder than observed (see Fig. 6). Core He-burning stars as old as $200 \mathrm{Myr}$ are crowding the clump of the red stars brighter than $F 814 W \sim 27$ and having $(F 555-F 814)<1.2$. We expect to have a good time resolution for ages younger than about $200 \mathrm{Myr}$. At this age, main sequence stars and/or He-burning loop stars are brighter than the completeness limit.

Concerning the presence of an older population, hints can be derived from the CMD. The large clump redder than $(F 555-F 814) \sim 1.2$ extending down to the photometry limit of $F 814 W \sim 27$ is consistent with the presence of AGB and He-burning stars of $Z=0.008$ in the age range 600-1000 Myr. Comparing the observational CMD with isochrones as metal-poor as $Z=0.0001$, it is evident that

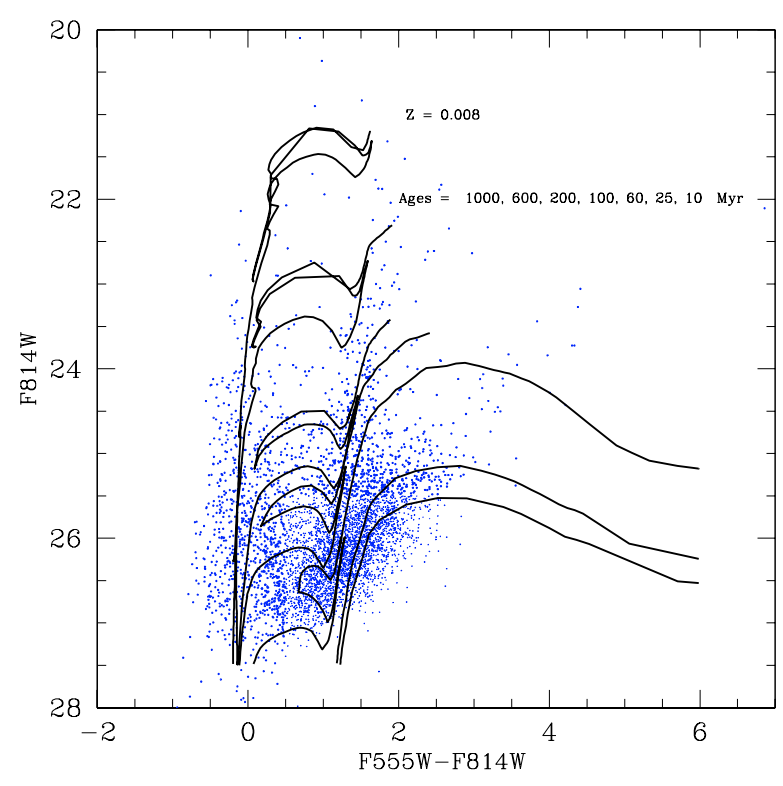

Fig. 5. CMD of UGC 5889 with isochrones of metallicity $Z=0.008$. For clarity, not all the stars in the field of UGC 5889 are plotted.

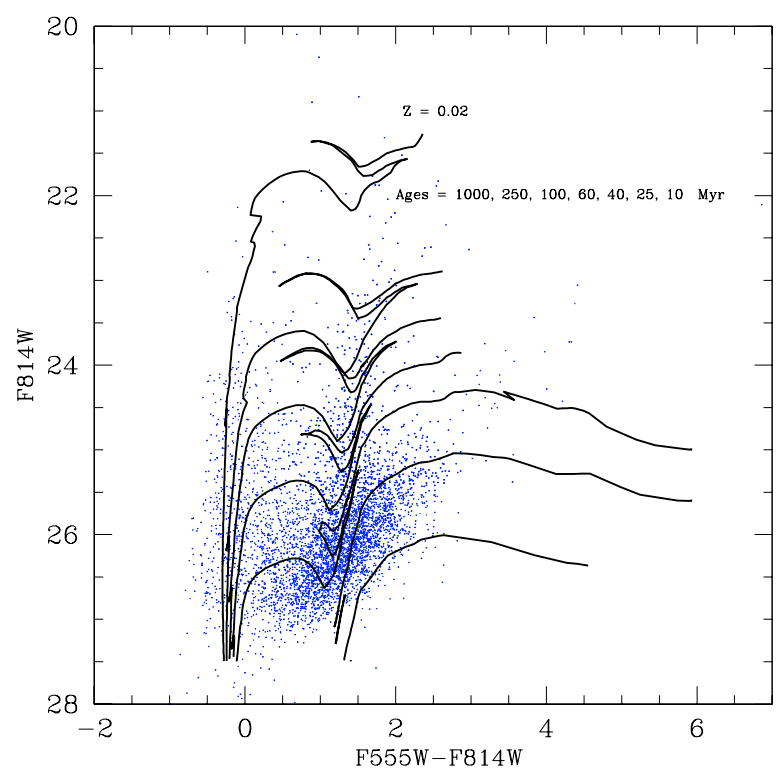

Fig. 6. CMD of UGC 5889 with isochrones of metallicity $Z=0.02$.

this metallicity cannot reproduce the AGB/RGB colour of stars younger than 1 Gyr (see Fig. 7). However, the existence of a metal poor population $(Z \leq 0.0001)$ in the age range $1-12$ Gyr cannot be ruled out. Stars of this age in the RGB/AGB phase are expected to appear at magnitudes fainter than $F 814 W \sim$ 23.5 and at a colour $(F 555-F 814)>1.2$.

\section{Star formation rate}

In order to infer the SFR of this galaxy, theoretical CMDs in different age ranges are simulated. These include the spread due to observational photometric errors. For each age bin, 1000 stars were generated. The generation of the synthetic populations makes use of the set of stellar tracks by Girardi et al. (1996, 2000). The initial mass function (IMF) of 


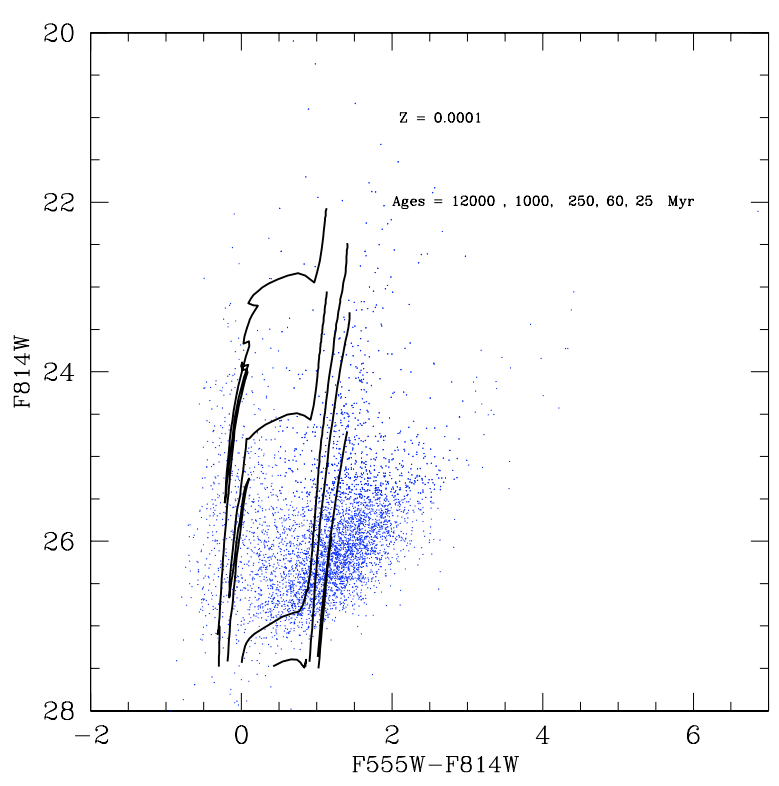

Fig. 7. CMD of UGC 5889 with isochrones of metallicity $Z=0.0001$.

Kroupa $(2001,2002)$ is assumed. This IMF is a power-law function with a slope $\alpha=2.3$ for stellar masses $m>0.5 M_{\odot}$, while $\alpha=1.3$ in the mass range $0.08-0.5 M_{\odot}$ (when the standard Salpeter value is 2.35). The constancy of the IMF slope in different environments is still a matter of discussion, although a number of recent papers proposed the idea of a universal IMF (see e.g. Kroupa 2002; Wyse et al. 2002; Chabrier 2003; Weidner \& Kroupa 2004). Recently, it has been suggested that $\alpha$ might depend on the galaxy type. For the IMF in LSB galaxies, it has been proposed that the effect of the low metal content can result in an inefficient SF taking place mainly outside the giant molecular clouds, thus yielding an IMF deficient in massive stars. As a consequence the IMF slope might be as steep as $\alpha \sim 3.8$ (Schombert et al. 1990). However, in this paper a more conservative approach is used, since a variation of the IMF slope in LSB galaxies is not at present completely justifiable (Lee et al. 2004). The completeness of the data is taken into account by dividing the simulated CMD in magnitude-colour bins and then subtracting from each bin having $N_{\text {th }}$ stars, $(1-\Lambda) N_{\text {th }}$, where $\Lambda$ is the smallest of the $F 555 W$ and $F 814 W$ completeness factors as given in Fig. 2.

Finally, the SFR is derived by means of a downhill simplex method (Harris \& Zaritsky 2001; Rizzi et al. 2002), minimising the $\chi^{2}$ function in a parameter-space having $N$ dimensions. At each step the local $\chi^{2}$ gradient is derived and a step in the direction of the gradient is taken, until a minimum is found. In the following, the observational CMD is divided into bins. Recent work concerning the determination of the SFR from the CMDs has pointed out the importance of using binning that takes into account the various stellar evolutionary phases, as well as the uncertainties on the stellar models (Rizzi et al. 2001). For this reason, while a coarser magnitude bin distribution is used, only two bins in colour are considered, namely from -0.5 to 0.5 , corresponding to blue plume stars, and from 0.5 to 3 , where the evolved stars are located. This avoids that the uncertainties on both the observational errors and the theoretical

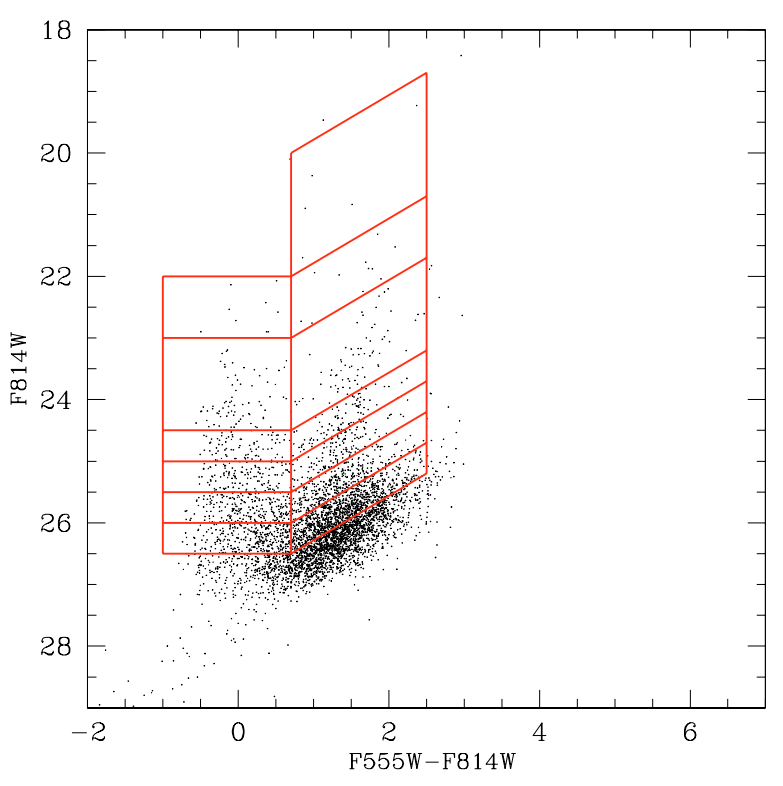

Fig. 8. The subdivision of the CMD into bins.

models (i.e. on bolometric corrections, RGB and AGB location, extension of the core He-burning loop) result in spurious solutions. Additionally the magnitude bins are taken in the blue part of the CMD at constant $F 814 W$ magnitude, while in the red part they are at constant $F 555 \mathrm{~W}$ magnitude. The reason for this is that the completeness limit in the red part of the CMD is mainly dependent on the completeness of the $F 555 \mathrm{~W}$ magnitude. This is illustrated in Fig. 8. To prevent settling on local rather than global minima, the simplex is first started from a random position, then when a possible solution is obtained, it is re-started from a position very close to it. Finally, when a minimum is found, 30000 random directions are searched for a new minimum.

The first guess solution is obtained by comparing the observational CMD with isochrones of different ages and metallicities (see previous section). We use $N=14$ stellar populations, whose ages are listed in Table 2. The chemical enrichment law is assumed as a pre-defined input parameter, derived comparing data and isochrones. Following the discussion of the previous section for ages younger than $20 \mathrm{Myr}$, we assume that the stars are generated with a metal content randomly chosen in the range $Z=0.004-0.01$. SFR episodes in the age range $20 \mathrm{Myr}-200 \mathrm{Myr}$ are assumed to be produced by stars having $Z=0.001-0.01$. An older population in the age range $0.2-1$ Gyr with the same metal content is included. A population of metal-poor stars with $Z=0.0003-0.004$ and as old as $1-12$ Gyr is also taken into account, since due to the low metal content, their magnitudes will be brighter than the photometry limit of $F 555 W \sim 27.5$. Metal-rich stars in the same age range are not considered since they are expected to be too faint. As discussed in Sect. 4, we are aware that we have poor age and SF resolution for populations older than $200 \mathrm{Myr}$. Therefore, the old age bin is included only to get an indication of the presence of an old population.

In Fig. 9 the best solution is plotted. The corresponding best fit SFRs are given in Table 2. We derive a $\chi^{2}$ value of 1.7. 

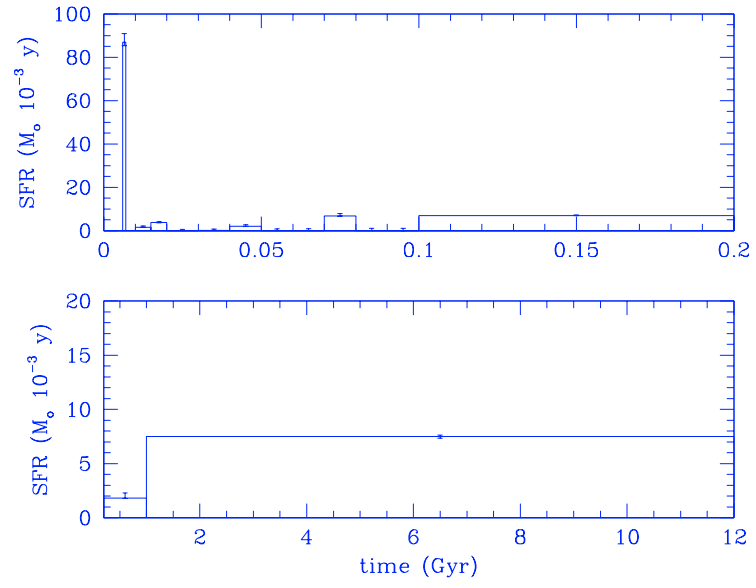

Fig. 9. SFR of UGC 5889 as a function of look-back time. Note that due to the low age resolution beyond $1 \mathrm{Gyr}$, we only give the average SFR in the lower plot (see text and Table 2).

Table 2. The solution parameter.

\begin{tabular}{c|ccccc}
\hline \hline & $t_{\text {in }}$ & $t_{\text {fin }}$ & $S F R$ & $S F R_{\text {low }}$ & $S F R_{\text {high }}$ \\
& $G y r$ & $G y r$ & $10^{-3} M_{\odot} / \mathrm{y}$ & $10^{-3} M_{\odot} / \mathrm{y}$ & $10^{-3} M_{\odot} / \mathrm{y}$ \\
\hline SFR1 & 0.006 & 0.007 & 86.9 & 85.3 & 90.9 \\
SFR2 & 0.010 & 0.015 & 1.6 & 1.6 & 2.1 \\
SFR3 & 0.015 & 0.020 & 3.7 & 3.6 & 4.2 \\
SFR4 & 0.020 & 0.030 & 0.0 & 0.0 & 0.6 \\
SFR5 & 0.030 & 0.040 & 0.17 & 0.17 & 0.8 \\
SFR6 & 0.040 & 0.050 & 2.0 & 1.7 & 2.8 \\
SFR7 & 0.050 & 0.060 & 0.0 & 0.0 & 0.9 \\
SFR8 & 0.060 & 0.070 & 0.0 & 0.0 & 1.0 \\
SFR9 & 0.070 & 0.080 & 6.9 & 6.7 & 7.9 \\
SFR10 & 0.080 & 0.090 & 0.0 & 0.0 & 1.1 \\
SFR11 & 0.090 & 0.100 & 0.0 & 0.0 & 1.1 \\
SFR12 & 0.100 & 0.200 & 7.0 & 6.9 & 7.3 \\
SFR13 & 0.200 & 1.000 & 1.8 & 1.7 & 2.2 \\
SFR14 & 1.000 & 12.000 & 7.5 & 7.3 & 7.6 \\
\hline
\end{tabular}

In order to better understand the quality of the fit, we compare the values of $\chi^{2}$ from two simulations with the same observational errors and input parameters, but different random number generators. The resulting $\chi^{2}$ for the second solution is 1.5 and supports the original finding. The range of SFR corresponding to $68 \%$ of the confidence level is given in Table 2 . This formal error is obtained by exploring the parameter space by varying all the amplitudes simultaneously in random directions, stepping away from the minimum until the $\chi^{2}$ value indicates that we have reached the $68 \%$ confidence level. The number of random directions to be checked has been tested on the simulations. About 50000 random directions are explored and are found to be sufficient to derive the confidence level.

The recent star formation appears to have proceeded in modest episodes at a rate of the order of $10^{-2} M_{\odot} / y r$, with periods of lower star formation or even quiescence. A very short burst took place $6 \mathrm{Myr}$ ago and lasted about $1 \mathrm{Myr}$. Any other solution involving different durations, intensities, or age scales

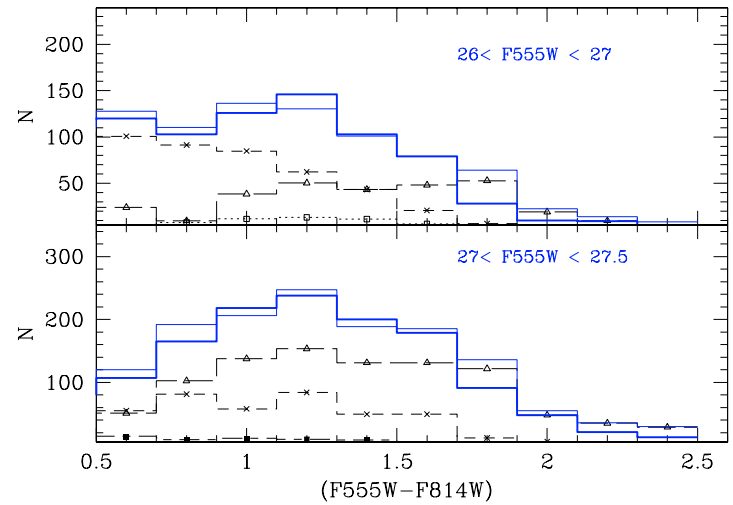

Fig. 10. The observational colour distribution (heavy solid line) compared with the simulations (thin solid line) in two magnitude bins when the population 13 older than $1 \mathrm{Gyr}$ is taken into account. Open squares indicate the simulated colour distribution in the age range 0.09-0.1 Gyr, crosses are the analogous values in the age range $0.1-0.2$ Gyr. Filled squares show the analogous values in the age interval 0.2-1.0 Gyr. Open triangles represent the simulated distribution for ages older than $1 \mathrm{Gyr}$.

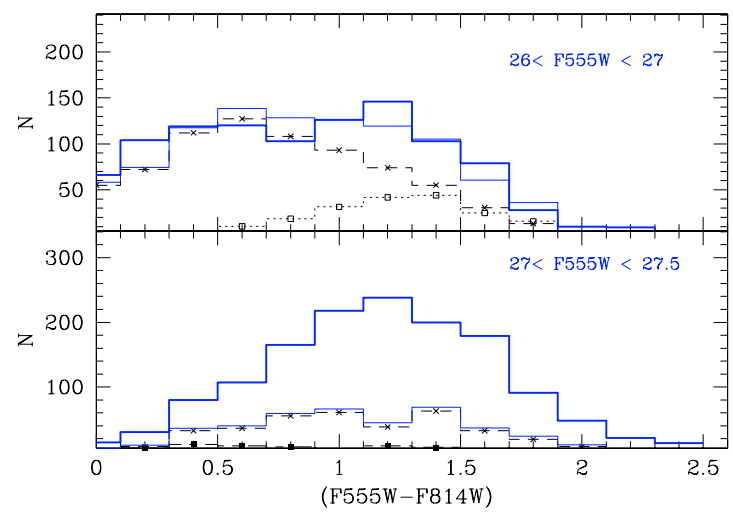

Fig. 11. The observational colour distribution (heavy solid line) compared with the simulations (thin solid line) in two magnitude bins when the population 13 older than $1 \mathrm{Gyr}$ is not included. Open squares indicate the simulated colour distribution in the age range $0.09-0.1 \mathrm{Gyr}$, crosses are the analogous values in the age range $0.1-0.2$ Gyr. Filled squares show the analogous values in the age interval $0.2-1.0 \mathrm{Gyr}$.

for star formation episodes younger than $10 \mathrm{Myr}$ yields a worse fit.

To assess the reliability of the solution at old ages, the downhill simplex method is applied to a different sets of initial ages, excluding in turn the oldest populations, in the age range $0.2-1$ Gyr and 1-12 Gyr, respectively. The simplex converges at high values of $\chi^{2}, 4.6$ and 2.9 respectively. To better verify the presence of an old population, we consider the CMD stars redder than $(F 555 W-F 814 W) \sim 0.5$ and fainter than $F 555 W \sim 26$. This region of the CMD is populated by stars older than 0.09 Gyr. We analyse the colour distribution in two magnitude bins, namely $26<F 555 W<27,27<F 555 W<$ 27.5 , corresponding to the two faintest magnitude bins in the red part of the CMD in Fig. 8. We make use of the last 4 populations of Table 2. Figures 10 and 11 present the best fit solution together with the data in two cases, a) including the 


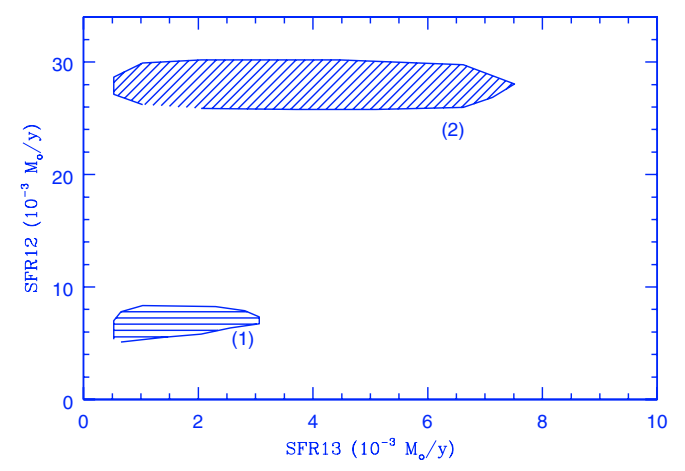

Fig. 12. The confidence interval for the SFR of population 12 and 13 derived from the red stars in the faintest magnitude bins, namely $26<$ $F 555 W<27$ (1), $27<F 555 W<27.5$ (2), when no population older than $1 \mathrm{Gyr}$ is included in the data (see text for details).

population older than $1 \mathrm{Gyr}$, and b) without it. In the case b) it is not possible to fit all the magnitude bins with the same combination of populations. For instance in Fig. 11 the SFR fitting the bin 27-27.5 cannot reproduce the data in the brighter magnitude bins. This is evident as well from Fig. 12 where the confidence interval for the SFRs of populations 12 and 13 are plotted in case b). No common solution is found when the population older than $1 \mathrm{Gyr}$ is not included. However, taking into account the presence of observational errors and completeness correction, we prefer to conclude than the resolution of the data is sufficient to assess the presence of a consistent population older than $200 \mathrm{Myr}$, but finer age division cannot be reliably derived. Finally, the stability of the solution against different sub-divisions of the CMD in cells is tested: slightly larger uncertainties result when a constant bin of $0.5 \mathrm{mag}$ is used.

Figure 13 presents the simulated CMD for the best solution. The simulated CMD is similar to the observational one. The total mass going into stars is about $5.5 \times 10^{7} M_{\odot}$. Since the total mass of the gas at present is observationally estimated to be $1.6 \times 10^{7} M_{\odot}$ (Simpson \& Gottesman 2000), the present day gas fraction comes out to be $f_{\mathrm{g}}=M_{\mathrm{gas}} /\left(M_{\mathrm{gas}}+M_{\mathrm{stars}}\right)=$ 0.2 . An independent check of this value can be derived if $f_{\mathrm{g}}$ is expressed in terms of observable quantities as:

$f_{\mathrm{g}}=\left(1+M_{\mathrm{stars}} / L_{B} \times L_{B} / \eta M_{\mathrm{HI}}\right)^{-1}$.

Bell et al. (2003) derive for a Salpeter IMF:

$\log \left(M_{\text {stars }} / L_{B}\right)=1.737(B-V)-0.789$.

Using $(B-V)=0.64, M_{\mathrm{HI}} / L_{B}=0.3$, and $\eta=1.4$ we find $f_{\mathrm{g}}=0.17$ in agreement with the determination obtained from the SF.

\subsection{Star formation rate from $H_{\alpha}$ luminosity}

The total luminosity $L_{\mathrm{H}_{\alpha}}$ emitted in $\mathrm{H}_{\alpha}$ can be used to estimate the recent SFR. Following Kennicutt et al. (1994) the SFR can be obtained from

$S F R=1.26 \times 10^{-41} \times L_{\mathrm{H}_{\alpha}} / 10^{38} M_{\odot} \mathrm{yr}^{-1}$.

Adopting $L_{\mathrm{H}_{\alpha}} \sim 2.6 \times 10^{38} \mathrm{erg} \mathrm{s}^{-1}$ as derived for UGC 5889 by Knezek et al. (1999), we find $S F R \sim 4.0 \times 10^{-3} M_{\odot} \mathrm{yr}^{-1}$. This

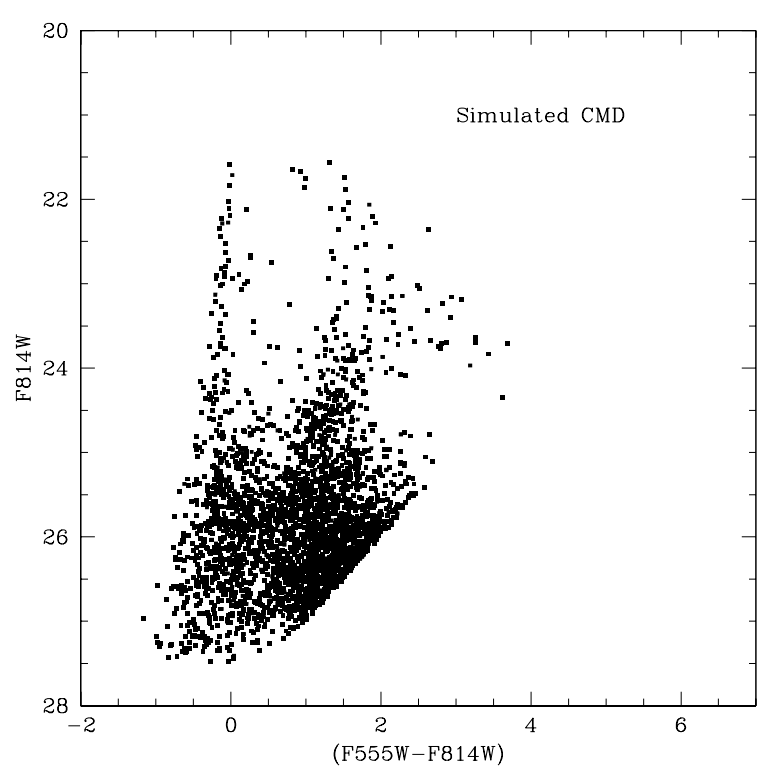

Fig. 13. The simulated CMD using the SFR of Table 2.

SFR should be regarded as the average value over the lifetime of the stars emitting in $\mathrm{H}_{\alpha}$. Assuming that the majority of the flux in $\mathrm{H}_{\alpha}$ is due to stars more massive than $10 M_{\odot}$, this gives an estimate of the SFR over the lifetime of a $10 M_{\odot}$ star. This lifetime can be derived from Girardi et al. (2000) tracks for $Z=0.008$ as $20 \mathrm{Myr}$. This SFR can be directly compared with the rate derived from the star-counts. Using the coefficients of Table 2, we derive a SFR of $5.6 \times 10^{-3} M_{\odot} \mathrm{yr}^{-1}$ averaged over the past $20 \mathrm{Myr}$ of the galaxy lifetime, in good agreement with the $L_{\mathrm{H}_{\alpha}}$ determination.

\subsection{The feedback from star formation}

The HI distribution of UGC 5889 shows a concentration of the gas in a ring surrounding the bulk of the optical body (Simpson $\&$ Gottesman 2000). The velocity field reveals a rotation of the entire ring-shaped distribution and no sign of expansion of the ring is found. This kind of disturbed interstellar medium is not unusual in dwarf galaxies (Puche \& Westphal 1993; Young \& Lo 1996; Simpson \& Gottesman 2000). The hypothesis naturally arises that this dramatic morphology might be the result of a vigorous star formation episode in a galaxy with a shallow potential well. In fact the energy injected in the gas by SNs and stellar winds can trigger the formation of super-bubbles that can easily expand in a low density medium. The expansion sweeps up the gas, forms a cavity at the centre, and leaves behind a system where the majority of the gas is located in the outskirts. This gas can be lost by the galaxy or can cool and rain back on to the galaxy after a cooling time (McLow \& Ferrara 1999). In this section, we discuss whether the energy injected in the gas by the SN explosions we estimate on the basis of the derived SFR can be sufficient to explain the morphology of the HI in UGC 5889. Finally, we discuss what might be the fate of this gas, i.e. whether a blow-out or a blow-away can take place. Following McLow \& Ferrara (1999) and Ferrara \& Tolstoy (2000) we define a blow-out as a process where the central SN explosions carve a hole in the gas disk, accelerating 
a fraction of it, while the remaining gas stays almost unperturbed. In a blow-away nearly all the gas is accelerated above the escape velocity and is lost from the galaxy.

To discuss the possibility of blow-out, let us assume that, perpendicular to the galactic plane, the unperturbed gas density distribution in the galaxy is exponential $\rho(z)=\rho_{0} \exp (-z / H)$, where $z$ is the vertical coordinate, $H$ is the galactic exponential scale height. The velocity of the shock wave produced by the $\mathrm{SN}$ explosions can be expressed as $v \sim(p / \rho)^{(1 / 2)}$, where $\mathrm{p}$ is the pressure. The velocity $v$ of the shock produced in a stratified medium decreases down to a minimum occurring at $z=3 \mathrm{H}$ before being re-accelerated in case of a blow-out. A blow-out takes place when the velocity exceeds the escape velocity of the galaxy at $z=3 H$, where the shock wave is accelerated to infinity. LSB galaxies are believed to be dark matter dominated (McGaugh \& de Block 1998). In order to calculate the escape velocity, the galaxy is modelled including the presence of a dark matter halo as in Persic \& Salucci (1996). The dark to visible mass ratio $\phi$ can be expressed as a function of the visible galaxy mass $M_{\mathrm{g}, 7}$ in $10^{7} M_{\odot}$, as

$\phi \sim 34.7 M_{\mathrm{g}, 7}^{-0.29}$.

Assuming as $M_{\mathrm{g}, 7}=19$ (Simpson \& Gottesman 2000), we find $\phi \sim 14$. This value is in reasonable agreement with the ratios in the range 10-30 derived for LSB galaxies by McGaugh \& Blok (1998). The blow-out condition can be expressed as (McLow \& Ferrara 1999):

$L_{38}>1.2 \times 10^{-2} M_{\mathrm{g}, 7} c_{10}^{2} h \phi /(\phi+1)$

where $L_{38}$ is the mechanical luminosity of the $\mathrm{SN}$ explosions i.e. the energy produced by $\mathrm{SN}$ explosions divided by the average lifetime of the less massive star $m_{\text {low }}$ exploding as a SN. If we assume $m_{\text {low }} \sim 10 M_{\odot}$ then its lifetime is 20 Myr. $L_{38}$ is expressed in $10^{38} \mathrm{erg} \mathrm{s}^{-1}$ when $c_{10}=1$ is the sound speed of the interstellar medium in $10 \mathrm{~km} \mathrm{~s}^{-1}$ including a turbulence contribution, and $h$ is the scaled Hubble constant, assumed to be 0.65. Comparing the HI location with the optical image, it is clear that the youngest stars are located in the outskirts of the gas distribution. Hence, the youngest star formation episode at $6 \mathrm{Myr}$ cannot have induced the $\mathrm{SN}$ explosions responsible for the central gas depletion. For this reason, we calculate $L_{38}$ using the mean SFR derived from our model in the last $20 \mathrm{Myr}$. Substituting in the blow-out condition we obtain:

$L_{38} \sim 6 \gg 0.14$.

This means that the blow-out condition holds true inside the gas: a blow-out is then responsible for the peculiar morphology of the ISM in UGC 5889. By definition, the blow-out involves only a fraction of the mass of the gas, that which is inside the cavities created by the SN explosions. A more disruptive phenomenon is the blow-away in which the gas content of the parent galaxy is completely lost. The blow-away can take place when the momentum of the gas shell at the radius $r_{\mathrm{s}}$ is larger than the momentum necessary to accelerate the gas outside $r_{\mathrm{s}}$ at a velocity larger than the escape velocity. This condition can be expressed following McLow \& Ferrara (1999) as:

$L_{38}>8 \times 10^{-2} M_{\mathrm{g}, 7}^{4.9}\left(\phi / \omega_{\mathrm{o}}\right)^{6} c_{10}^{-10} h$ where $\omega_{0}=3$ is a scale factor. Under reasonable assumptions concerning the sound speed $c_{10}=1.5-0.5$, this condition is not verified even if $\phi$ is assumed to vary in the range 5-30. The ultimate fate of the gas seems to be that it will not leave its parent galaxy. This result is in full agreement with the low gas velocity derived observationally (Simpson \& Gottesman 2000).

\section{Comparison with other LSB galaxies}

Bursty star formation history in LSB galaxies has been suggested in the literature. We quote, from amongst others, Boissier et al. (2003) who find that it is necessary to include star formation events such as bursts in the models of LSB galaxy evolution to account for the observed scatter in the Tully-Fisher and total mass of the gas-to-luminosity relation. A burst scenario is proposed by O'Neil et al. (1998) to explain both the blue colour as well as the disrupted morphology of most blue LSB galaxies.

The dwarf irregular galaxy IC 1613 has similar colours $(B-V=0.71)$ and its SF has been studied in great detail. Cole et al. (1999) find a nearly constant SFR at a low level of $1.6 \times$ $10^{-3} M_{\odot} \mathrm{yr}^{-1} \mathrm{kpc}^{-2}$ over the past $1-300 \mathrm{Myr}$. However, due to the large angular size of IC 1613, they only study the very central part of it, which might not be representative of the SF over the whole galaxy. Note for example that in UGC 5889, the recent SF is concentrated on the outer edge, while the centre is inhabited by the older population. Skillman et al. (2003) compared it with a field situated at the outer edge of IC 1613, but studied the intermediate-age and ancient SFR, starting at an age of 100 Myr. A comparison with the CMD of Cole et al. yielded no significant difference. Although their data are consistent with constant SFR, they find small bursts in the SFR and a strong enhancement of the SFR at intermediate ages. However, no evidence is found for a strong, bursty SFR in the recent history. In fact, the CMDs presented by Skillman et al. as well as the one by Cole et al. lack the bright blue stars that are present in UGC 5889 and can only be explained by recent SF. Therefore, although both galaxies are classified as blue LSB galaxies they differ in their SFR in the sense that IC 1613 is dominated by SF at intermediate age, while UGC 5889 is dominated by more recent SF. This behaviour is also reflected by the total mass-over-luminosity ratio, which is slightly lower for UGC 5889.

\section{Conclusions}

In this paper we have discussed the star formation history of the LSB galaxy UGC 5889 on the basis of HST archive data. Our main conclusions can be summarised as the following:

1) The RGB tip is detected at $F 814 W=25.6 \pm 0.2$ implying a distance modulus of $(m-M)_{0}=29.4 \pm 0.2$.

2) The recent star formation is proceeding in modest bursts at a rate of the order of $10^{-2}-10^{-3} M_{\odot} / \mathrm{yr}$. This rate is lower than the $5-10 M_{\odot} / \mathrm{yr}$ derived for high surface brightness disk galaxies (Kennicutt 1992), but only slightly larger or comparable to the $10^{-3} M_{\odot} /$ yr observed in dwarf irregulars (Hunter \& Gallagher 1986). The SFR derived in UGC 5889 
from $\mathrm{H}_{\alpha}$ emission is in agreement with the results obtained from CMD analysis.

3) The existence of a consistent population older than $200 \mathrm{Myr}$ and possibly older than 1 Gyr is suggested by our analysis. However, this result might depend on the completeness correction and observational errors and needs to be verified with deeper photometry.

4) The present-day total mass in stars is of the order of $5.5 \times$ $10^{7} M_{\odot}$. Since the total mass of the gas has been estimated as $1.6 \times 10^{7} M_{\odot}$, the gas fraction is $f_{\mathrm{g}}=0.2$.

5) The morphology of the gas inside this galaxy reveals a large cavity, including the main optical body of the galaxy. The gas is mainly located in the external regions. We have calculated the SFR feed-back on the interstellar gas. We find that this impressive morphology is consistent with the hypothesis that $\mathrm{SN}$ explosions have produced a blow-out of the gas from the internal regions towards the external ones. On the basis of our calculations, it seems however quite unlikely that the final fate of this gas is to leave the galaxy.

Acknowledgements. This research has made thorough use of the Simbad database operated at CDS, Strasbourg, France. This work was done while A.V. was visiting scientist at ESO, Santiago. We thank the referee, P. Knezek, whose comments greatly improved this paper. We thank K. O'Brien for a careful reading of the manuscript. We are indebt to G. Bertelli for many fruitful discussions.

\section{References}

Barker, M. K., Sarajedini, A., \& Harris, J. 2004, ApJ, 606, 869

Bell, E. F., \& de Jong, R. S. 1999, MNRAS

Bell, E. F., McIntosh, I. H., Katz, N., \& Weinberg, M. D. 2003, ApJS, 149,289

Bellazzini, M., Ferraro, F. R., \& Pancino, E. 2001, ApJ, 556, 635

Bellazzini, M., Ferraro, F., Sollima, A., Pancino, E., \& Origlia, L. 2004, A\&A, 424, 199-211

Bergmann, M. P., Jorgensen, I., \& Hill, G. 2003, AJ, 125, 116

Boissier, S., Monniere Ragaigne, D., Prantzos, N., et al. 2003, MNRAS, 343, 653

Bressan, A., Fagotto, F., Bertelli, G., \& Chiosi, C. 1993, A\&AS, 100, 647

Chabrier, G. 2003, PASP, 115, 763

Cole, A. A., Tolstoy, E., Gallagher, J. S., et al. 1999, ApJ, 118, 1657

de Blok, W. J. G., \& Van der Hulst, J. M. 1998, A\&A, 335, 421

Dalcanton, J., Spergel, D., \& Summers, F. 1997, ApJ, 482, 659

Diplas, A., \& Savage, B. D. 1994, ApJ, 427, 274

Dolphin, A. E. 2000, PASP, 112, 1397

Ferrara, A., \& Tolstoy, E. 2000, MNRAS, 313, 291

Gallagher, J. S., Tolstoy, E., Dohm-Palmer, R. C., et al. 1998, AJ, 115 , 1869

Girardi, L., Bressan, A., Chiosi, C., Bertelli, G., \& Nasi, E. 1996, A\&AS, 117, 113

Girardi, L., Bressan, A., Bertelli, G., \& Chiosi, C. 2000, A\&AS, 141, 371

Graham, J. A., Phelps, R. L., Freedman, W. L., et al. 1997, ApJ, 477, 535

Harris, J., \& Zaritsky, D. 2001, ApJS, 136, 25

Hartmann, D., \& Burton, W. B. 1997, Atlas of Galactic Neutral Hydrogen (Cambridge: Cambridge Univ. Press)
Huchra, J. 1977, ApJS, 35, 171

Huchtmeier, W. K., Karachentsev, I. D., \& Karachentseva, V. E. 2003, A\&A, 401, 483

Hunter, D. A., \& Gallagher, J. S. 1986, PASP, 98, 5

Kennicutt, R. C. 1992, ApJ, 388, 310

Impey, C., \& Bothun, G. 1997, ARA\&A, 35

Kennicutt, R. C., Tamblyn, P., \& Congdon, C. E. 1994, ApJ, 435, 22

Knezek, P. M. 1993, Ph.D. Thesis, University of Massachussets

Knezek, P. M., Sembach, K. R., \& Gallagher, J. S. III 1999, ApJ, 514, 119

Kroupa, P. 2001, MNRAS, 322, 231

Kroupa, P. 2002, Science, 295, 88

Kroupa, P., Tout, C. A., \& Gilmore, G. 1993, MNRAS, 262, 545

Lee, M. G., Freedman, W. L., \& Madore, B. F. 1993, ApJ, 417, 553

Lee, H., Gibson, B. K., Flynn, C., Kawata, D., \& Beasley, M. A. 2004, MNRAS, 1365, 246

Makarova, L. N., \& Karachentsev, I. D. 1998, A\&AS, 133, 181

Marzke, R. O., \& da Costa, L. N. 1997, AJ, 113, 185

McGaugh, S. S., \& Blok, W. J. G. 1997, ApJ, 481, 689

McGaugh, S. S., \& Blok, W. J. G. 1998, ApJ, 499, 41

McLow, M. M., \& Ferrara, A. 1999, ApJ, 513, 142

Ng, Y. K., Bertelli, G., Bressan, A., Chiosi, C., \& Lub, J. 1995, A\&A, 295, 655

O’Neil, K., \& Bothun, G. 2000, ApJ, 529, 811

O’Neil, K., Bothun, G. D., \& Schombert, J. 1997, AJ, 114, 2448

O’Neil, K., Bothun, G., \& Schombert, J. 1998, AJ, 116, 2776

O’Neil, K., Verheijen, M. A. W., \& McGaugh, S. S. 2000, AJ, 119, 2154

Padoan, P., Jimenez, R., \& Antonuccio-Delogu, V. 1997, ApJ, 481, L27

Persic, M., \& Salucci, P. 1996, in Non-Accelerator Particle Physics, Proceedings of the Fourth School, held 17-28 July, 1995 at International Centre for Theoretical Physics, Trieste, Italy, ed. E. Bellotti, R. A. Carrigan, Jr., G. Giacomelli, \& N. Paver (Singapore: World Scientific), 79

Prugniel, P., \& Heraudeau, P. 1998, A\&AS, 128, 299

Puche, D., \& Westfahl, D. 1994, IN ESO/OHP Workshop on Dwarf Galaxies, ed. G. Meylan, \& Prugniel (Garching, ESO), 273

Rizzi, L., Held, E. V., Bertelli, G., et al. 2002, in Observed HR Diagrams and Stellar Evolution, ed. T. Lejeune, \& J. Fernandes (San Francisco: ASP), ASP Conf. Proc., 274. 490

Sandage, A., \& Hoffman, G. L. 1991, ApJ, 379, L45

Schlegel, D. J., Finkbeiner, P., \& Davis, M. 1998, ApJ, 500, 525

Schombert, J. M., Bothun, G. D., Impey, C. D., \& Mundy, L. G. 1990, AJ, 100, 1523

Simpson, C. E., \& Gottesman, S. T. 2000, AJ, 120, 2975

Skillman, E. D., Tolstoy, E., Cole, A. A., et al. 2003, ApJ, 596, 253

Sprayberry, D., Impey, C. D., Irwin, M. J., \& Bothun, G. D. 1997, ApJ, 482, 104

Turner, A. 1996, Cooking with ALLFRAME

van den Hoek, L. B., de Blok, W. J. G., van der Hulst, J. M., \& de Jong, T. 2000, A\&A, 357, 397

van Zee, L., Haynes, M. P., \& Salzer, J. J. 1997, AJ, 114, 2479

Vicari, A., Battinelli, P., Capuzzo-Dolcetta, R., Wyder, T. K., \& Arrabito, G. 2002, A\&A, 384, 24

Weidner, C., \& Kroupa, P. 2004, MNRAS, 348, 187

Wyse, R. F. G., Gilmore, G., Houdashelt, M. L., et al. 2002, New Astron., 7, 395

Young, L. M., \& Lo, K. Y. 1996, ApJ, 462, 203 